Wir können also Mikrokokken und Stäbchenbakterien zwar für viele, aber keineswegs für alle Fälle die Bedeutung als Erzenger der Eiterung anerkennen.

Dagegen vermag auch eine intensiv wirkende chemische Ursache, zumal wenn eine mechanische Reizung damit verknüpft ist, ohne jede Mitwirkung niederer Organismen durch sich allein die heftigste Eiterung hervorzurufen.

\author{
VI. \\ Veber Krebsmetastasen im Magen. \\ Casuistiscbe Mittheilung. \\ Von Dr. Paul Grawitz, \\ Assistenten am pathologisehen Institute zu Berlin.
}

Der Magen ist, wie die Statistik nachweist, dasjenige Organ, welches am häufigsten von primärer Krebsbildung befallen wird; Marc d'Espine fand dieselbe unter 889 Carcinomfällen 399 mal, Lange, welcher die Sectionsprotocolle des Berliner pathologischen Instituts von 1859-1875 zusammenstellte, unter 587 Fällen 210mal, woraus sich ein Durchschnitt von etwa 41 pCt. unter 1476 Gesammtbeobachtungen ergiebt.

Dieser Frequenz gegenüber gebören secundäre Krebseruptionen im Magen zu den grössten Seltenheiten, so dass die zahlreichen Auszüge aus den hiesigen Protocollen, welche auf Virchow's Veranlassung in Berliner Dissertationen veröffentlicht worden sind, nur 3 zweifellose Fälle dieser Art anführen ${ }^{1}$ ), darunter denselben, wel-

1) J. Hecker, 34 ín pathol. Institut zu Berlin vorgekommene Fälle von Mastdarmbrebs. Berlin. Inaug.-Diss. 1868. - J. Petri, 44 Falle von Krebs der Speiseröhre. Diss. Berlin 1868. Enthält 2 Fälle von metastatischem Magenkrebs. - B. Riesenfeld, 69 Fälle von Leberkrebs. Diss. Berlin 1868. Leop. Lange, Der Magenkrebs und seine Metastasen. Diss. Berlin 1877. F. Erich, Carcinoma mammae und seine Metastasen. Diss. Berlin 1877. S. Sommerfeld, Krebs der Brustdrüse. Inaug,--Diss. Berlin 1878. (Hier sind 2 Fälle angegeben, während Ericb ein Jahr früher our den von Cobn- 
chen Gohnheim im 38. Bd. dieses Archivs ausführlicher mitgetheilt hat. In einer Statistik von Coupland (Transact. of the pathol. Soc. of London 1876), welche 89 Fälle von Mammakrebs umfasst, figurirt unter den zahlreichen metastatisch erkrankten Organen der Magen nur 1mal, so dass es gewiss als eine Ausnahme und besondere Begünstigung $z$ betrachten ist, wenn ich über vier neue von mir beobachtete Krebsmetastasen berichten kann. Rechtfertigt also schon die geringe Zahl der bekannten secundären Magencarcinome an sich diesen casuistischen Beitrag, so füllt er noch mehr von einem zweiten Gesichtspunkte betrachtet eine gewisse Lücke aus. Es kommen nehmlich nicht ganz selten Befunde von allgemeiner Carcinosis vor, bei welchen der Magen einen kleinen flachen, wenig oder gar nicht ulcerirten Krebs enthält, der betreffs seiner unansehnlichen Grösse in einem solchen Missverhältniss zu den mächtigen Geschwälsten der Leber, der Lymphdrüsen und anderer Organe steht, dass auch dem erfahrenen Beurtheiler Zweifel aufsteigen, welche Neubildung als die primäre, und welche als die secundäre anzusehen ist. Solche Fragen können nur durch Vergleich mit zweifellos deuteropathischen Carcinomen des Magens geklärt werden, und eine Beschreibung solcher Fälle ist um so mehr Bedürfniss, als die Angaben der besten Lehrbüeher nicht auf eigenen Beobachtungen beruhen, und theilweise unrichtige Merkmale enthalten ${ }^{1}$ ). - Meine beiden ersten Fälle sind Metastasen primärer Oesophaguscarcinome.

Fall I. Julius Bogge, 49 Jahre alt, starb am 2. Nov. 1879. Die Díagnose der Section ergiebt Ulcus cancroides oesophagi; Phlegmone paroesophagealis apostematosa e perforatione carcinomatis. Carcinomata metastatica exulcerata et Ulcus simplex ventriculi. Degeneratio carcin. glandul. epigastric. Peribepatitis et Perigastritis adbaes. carcinomatosa. Phthisis chron. ulcerosa pulmonum. Atrophia fusca cordis et hepatis.

heim publicirten Fall aufgenommen hat. Es scheint hier ein Myomknoten irrthümlich als Metastase gezäblt worden za seín.)

1) So scbreibt Förster in seinem Handbuch S. 73: „Der Krebs kommt im Magen meist als primäre Entartung vor, während secundäre Krebsbildung zu den grössten Seltenheiten gehört, ich selbst habe nie einen solchen Fall gesehen, selbst in Leichen, in welchen fast kein Organ des Körpers von secundären Krebsknoten frei war; übrigens zeigte sich der secundäre Krebs im Magen in Form sparsamer oder zahlreicher umschriebener Knoten im submucösen Zellgewebe, welche keine weiteren Veränderungen eingeben." Klebs führt pur den Fall Cohnheim's an. 
Der Oesophagus zeigt nach Herausnahme der gleichmässig fest verwachsenen Langen nahe über der Durchtrittsstelle oberhalb des Zwerchfells eine jauchige Infiltration des umgebenden losen Bindegebes von $4 \mathrm{Cm}$. Länge, in deren Mitte grünlichgraue mit Bröckeln untermischte Flüssigkeit abgesackt ist, während dicke Schwielen der Pleuren den Uebertritt der Jauche in die Brustfellsăcke hindern. Bei der Herausnabme in toto erweisen sich Pharynx, Gaumen und die oberen zwei Dritttheile der Speiseröhre von glatter, unversehrter blassgrauer Oberfläche. Dann beginnt ein $6 \mathrm{Cm}$. langes, die ganze Circumferenz einnehmendes flaches krebsiges Geschwür mit sehr derber wallartiger Begrenzung und sehr unregelmässigem stellenweise tief buchtigem Grunde, in dessen Mitte eine für eine Fingerspitze durchgängige Perforationsöffnung in die erwähnte abgeschlossene Brandhöhle führt. Der unterste kaum $4 \mathrm{Cm}$. messende Abschnitt des Oesophagus ist in selner Innenfläche wiederum glatt und anscheinend normal, wäbrend das äussere, die Muscularis umkleidende Bindegewebe ziemlich derb infiltrirt ist. Diese krebsige Entartung erstreckt sich längs der epigastrischen Lymphdrüsenkette bis zur Porta hepatis fort, wo ansserdem vielfache fibröse Adhäsionen zwischen vorderer Magenwand und Leber durchtrennt werden müssen. Der Magen wird in seiner Portio cardiaca durch eine reichlich gänseeigrosse Geschwulstmasse eingenommen, die den Eingang zum Theil verlegt, and von sehr weichem Gefüge ist. Sie wird grösstentheils von granweisser Schleimhaut überzogen, nor im Centrum ist sie kraterförmig ulcerirt, ihre Rănder wallartig aufgeworfen. Sie durchsetzt alle Schichten der Magenwand, so dass auf einem Durchschnitt die bröcklige weisse Krebsmasse von der ulcerirten Schleimbaut continuirlich bis zu den geschwollenen und degenerirten Drüsen der kleinen Curvatur zn verfolgen ist. Unmittelbar neben diesem liegt an der kleinen Krümmung ein zweiter metastatischer Krebsknoten von dem Umfange eines Hübnereies, der gleichfalls die Serosa, Muscularis und Submucosa durchsetat, und auf der Höhe der Prominenz auch die Mucosa durchbricht, und exulcerirt ist. Wiederum einige Centimeter weiter zum Pylorus liegt seitlich der kleinen Curvatur ein etwa markstïckgrosses scharfrandiges, von glatter blassröthlicher Schleimhaut flach umgebenes rundes Magengeschwür, welches tief in die Muscularis hinabreicht. Seinem Sitz entsprechen die Verwachsungen mit der Leber.

Die mikroskopische Untersuchung ergiebt das gewöhnliche Bild eines Oesophaguscancroids mit grossen Perlkugeln in reichlicher Anzahl, dieselben Befunde auch in den metastatischen weichen Tumoren des Magens und des kleinen Netzes.

Fall II. Gottlieb Fischer, 59jähriger Billeteur am Circus Renz, aufgenommen am 14. Februar 1881 auf die propädeutische Klinik der Charité, starb am 24. Februar e. a. an janchiger Pleuritis, welche durch die Perforation eines krebsigen Geschwürs des Oesophagus entstanden war.

Diagnose. Ulcus carcinomatodes oesophagi. Phlegmone paroesophagealis e perforatione in cavum pleurae dextr. Pleuritis haemorrh. recens. Infiltr. carcinomatosa gland. bronchial, et epigastric. Carcinoma metastatium ventriculi et hepatis. Endocarditis chron. retrahens mitralis et aortica.

Die Section ergiebt eine mittelgrosse, sehr magere, männliche Leiche mit schmutzig grauwelsser Haut, sehr dünnem pigmentirten Fettpolster, schwach ent- 
wickelter atrophischer Musculatur. Der Stand des Zwerchfells entspricht beiderseits dem V. Intercostairaum. Das Herz ist von normaler Grösse, Pericard glatt und glänzend mit reichlicher Fettschicht auf der Oberfläche des rechten Vorhofes. Herzwănde dünn, schmutzig braun, sehr schlaff. Die Mitralis ist retrahirt, ihr vorderes Segel stark verdickt, die Sehnenfäden vielfach mit einander verschmolzen. Die Aortenklappen sind gleichfalls derb, in der Tiefe ibrer Taschen enthalten sie knorpelbarte mehrfach verkalkte Schwielen; an den Nodulis frischere Rauhigkeiten mit zarten thrombotischen Niederschlägen. Linke Lange frei beweglich, Oberfläche spiegelnd glatt, hellroth, Gewebe durchweg lufthaltig. Rechte Lunge zeigt bel der Herausnahme im unteren Abschnitt des stumpfen Randes eine Verwachsung mit der Speiseröhre, bei deren Trennung man auf eine janchig brandige Geschwürsstelle stösst, welche einem handtellergrossen Abschnitt der Pleura anliegt, der getrübt und von bämorrhagischen Infiltrationen umgeben ist. Pleura in weiterer Ungebung trübe, sammetartig rauh mit dünnem Fibrinbelag. Das Lungengewebe selbst ist von der Entzündung nicht ergriffen, sein Luftgehalt normal; am Hilus liegt eine beinahe taubeneigrosse krebsig entartete Lymphdrüse. Speiseröhre und Magen werden in toto herausgenommen. Dabei erweist sich der Rachen, weiche Gaumen und Larynx sebr anämisch, sonst unverändert. Der Oesophagus ist in seinen oberen 2 Dritttheilen glatt, rein weiss und zart. Unterhalb der Kreuzungsstelle mit dem linken Bronchus beginnt ein ca. $11 \mathrm{Cm}$. langes Geschwür, welches die ganze Circumferenz der Oesophaguswand einnimmt, und mit unregelmässiger Grenze etwa $4 \mathrm{Cm}$. oberhalb der Cardia abschneidet. Der Grund der Ulceration ist brandig zerfallen, es besteht elne Perforationsöffnung in die vorerwähnte, zwischen äusserer Oesophaguswand und Pleura gelegene abgekapselte Brandhöhle. Die Geschwürsränder sind callös verdickt, beim Einschneiden weiss, und lassen eine breiige Masse auspressen. Der onterste kurze Rest des Oesophagus ist wiederam intact. Im Magen, dicht hinter der Cardia liegt ein apfelgrosser, runder, $5 \mathrm{Cm}$. im Durchmesser betragender Geschwulstknoten, welcher von der kleinen Curvatur her die Schleimhant kugelig in den Magenraum vorwölbt, und daher der vom Oesophagus eindringenden Scheere einigermaassen den Weg verlegt. An der Kuppe dieses Tumors erscheint die Magenschleimhaut rosig roth und sehr verdünnt, an einer markstückgrossen Stelle ist sie von dem Tumor durchbrochen und oberflächlich ulcerirt. Der bei weitem grössere Theil der Magenwand ist etwas verdickt, seine Schleimhaut mässig geröthet. In dem Mageninhalt befindet sich eine Taenia solinm. Die Leber ist klein und blutreich, die Acini sind in ihren peripherischen Theilen fettinfiltrirt, die Centra pigmentirt; nahe dem Hilus liegt in dem Parenchym ein wallnussgrosser, weisser, bröcklig weicher Krebsknoten eingesprengt. Alle übrigen Organe sind ohne bemerkenswerthe Veränderungen.

Mikroskopisch erweist sich wie im 1. Falle die primäre Geschwulst des Oesophagus sowie die Metastasen in der Bronchialdrüse, im Magen und der Leber als ein Cancroid mit grossen platten, vielfach zu Perlkugeln zusammengeballten Zellen und sehr zartem anämischen Geriust. 
Die primäre Natur des Oesophaguskrebses unterliegt in beiden Făllen keinem Zweifel, wie die Stătte der Entwicklung, die Grösse der Neubildung, die mikroskopische Structur darthun; der Weg der Verbreitung ist beide Male in dem Lymphstrom zu suchen, der ja stellenweise per continuitatem die Verbindung mit dem Haupttumor hergestellt hat.

Der III. Fall, Carl Hoffmann, Fuhrherr, wurde im Juli 1879 dem Institut zar Untersuchung aus dem Augusta-Hospital übersandt.

Diagnose: Fungus carcinomatosus testis et Carcinomata metastatica ventriculi, pleurae pulmonalis utrq. et pulmonum, hepatis renum, Glandularum suprarenalium. Intestini, Gl. thyreoideae, testiculi alterius.

Der Hoden zeigt sich bei der Untersuchung stark vergrössert, in eine faustgrosse, äusserlich dunkelkirschrothe, ungemein weiche und brïchige Tumormasse umgewandelt, in welcher von dem normalen Gewebe nirgends mehr Spuren za bemerken sind. Auch der Nebenhoden ist in die Neubildung mit aufgegangen. Beide Lungen enthalten in der übrigens glatten und dünnen Pleura sehr zahlreiche, theils flache, theils kugelige Tumoren, welche von Linsengrösse bis zu Kirsch- und Hühnereigrösse alle Uebergänge darbieten. Sie sind von gleichmàssig dunkelrother Farbe, und von schwammig weicher Textur, beim Einschneiden entleert sich aus ibnen reichliches flüssiges Blut, während an anderen Abschnitten festere Blutklumpen im Gewebe stecken. Ein Durchschnitt durch die Lungen zeigt inmitten des hyperämischen und lufthaltigen Parenchyms mehrere apfelgrosse derbere Geschwülste von dem beschriebenen Aussehen. Der Magen ist weit, seine Wand im Allgemeinen dünn, die Schleimhaut von hellgraner, im Fundus leicht röthlicher Farbe. An der vorderen Wand, etwa auf der Hälfte zwischen Cardia und Pylorus wölbt sich ein pllaumengrosser kugeliger Tumor in das Lumen vor, der in seinen Randzonen von Schleimhaut bekleidet ist, in der Mitte aber eine zehnmarkstückgrosse tiefgreifende Verschwärung enthält, aus der sich blutiger Saft ausdrủcken lăsst. Der Knoten liegt in der Submucosa und der Drüsenschicht, die letztere in grossem Umfange durchwuchernd, über der Muscularis ist er ein wenig verschieblich. Nahe diesem ulcerirten Tumor liegt hart an der grossen Curvator ein zweiter kleinerer, rein submucös, von der Grösse einer Mandel. Ueber diesen zieht die Schleimhant zwar etwas verdünnt, aber doch glatt hinweg; er besteht aus demselben rothen schwammigen Gewebe wie die schon beschriebenen Bildungen des Hodens und der Lungen.

Mikroskopisch stellt sich beraus, dass sowohl in dem Haupttumor wie in den Metastasen ein medulläres zellenreiches Krebsgewebe entbalten ist, dessen rothes Aussehen theilweise von einer überreichen Bildung weiter kleiner Gefässe herrührt, theilweise aber auf wirkliche Hämorrhagien in das brüchige Geschwulstparenchym zu beziehen ist.

Dieses Carcinoma teleangiectodes haemorrhagicum hat nun seinen Primärsitz in dem Hoden, wie einmal die klinische Beob- 
achtung, dann aber die Grösse der Neubildung klar ergiebt. Bei der Multiplicität der Eruptionen in Pleura, Lunge und Magen würde selbst obne die Krankengeschichte eine Missdeutung nicht zu befürchten sein. Der Weg der Verbreitung lässt sich nicht mehr mit Bestimmtheit verfolgen, da nicht sämmtliche Metastasen übersandt wurden, und Notizen über die Beschaffenbeit der Gefässe fehlen, indessen ist auch hier wohl mit Wahrscheinlichkeit die Ansteckung durch den Lymphstrom vermittelt worden.

IV. Fall. Carcinoma mammae recidivum. Degeneratio carcin. gland. axillar. mediastinalium, cervicalium, epigastric. retroperit. Carcinomata metastat. cordis plearae, pulmonum, diaphragmatis, ventriculi, hepatis renum, gl. suprarenalium, ovariorum, vesicae urin. Nervorum cerebralium et spinalium.

Am 3. Juni 1881 verstarb auf der Irrenabtheilung der Charité die 69jährige Fran Emma Fromm. Derselben war 10 Monate früher auf der chirurgischen Universitätsklinik ein Carcinom der rechten Mamma exstirpirt worden. Da in der Umgebung der Narbe sowie in der rechten Axelhöhle mehrfache kirschgrosse, derbe Tumoren durch die Haut leicht durchzufühlen waren, da der Ernährungszustand der an chronischer Verrücktheit leidenden Frau auf allgemeine Krebskachexie bindeutete, und $d a$ in den letzten Lebenswochen Anzeichen centraler Lähmungen im Bereich der Nn. ciliares, des rechten Facialis und des rechten Hypoglossus sich zu den bis dahin schwer erklärlichen Gehstörungen hinzugesellten, so befestigte sich mehr und mehr die Vermuthung, dass es sich im vorliegenden Falle um eine secundäre Krebseruption an der Gehirnbasis und vielleicht auch im Rückenmarkskanale handeln würde. Die Section bestätigt nicht nur die Diagnose auf das Vollkommenste, sie ergab auch ausserdem eine solche Fülle von Geschwulstheerden in nahezu allen Organen, so dass er als besondere Rarität wohl eine ausfübrlichere Beschreibung verdienen mag.

Sectionsprotocoll: Weibliche Leiche von mittlerer Grösse, kräftiger Statur in sehr dürftigem Ernäbrungszustand. Die Haut von schmutzig weisser Farbe, das Fettgewebe sehr dünn, gelbbraun pigmentirt, die Muskeln schlaff und welk blassroth, die Todtenstarre gelöst. Die Gegend der rechten Mamma wird von einer handtellergrossen flachen weissen Narbe eingenommen, unter der vom M. pectoralis nichts mehr wahrzunehmen ist. In der Umgebung der Narbe gewahrt man sowohl nach dem Rippenrande und Schwertfortsatze als besonders nach der Axel hin mebrere erbsen- bis kirschgrosse, ziemlich derbe Knoten, welche theilweise unter der Haut verschieblịch, theils mit ihr fest verwachsen sind. Sie sind beim Einschneiden grauweiss; auf Druck entleert sich ein ziemlich reichlicher Milchsaft. Im Uebrigen ist an der Haut nichts von Geschwolstbildungen zu bemerken; auch das Knochensystem erscheint frei davon. Das Abdomen ist tief eingesunken. Nach seiner Eröffnung sieht man das anämische fettarme Netz über die engen, röthlich grauen oder schmutzig schiefergrauen Darmschlingen bis zum Beckeneingange ausgebreitet; der Peritonealüberzug ist überall glatt und glänzend, im abhängigen Theil der Bauchböhle befinden sieh ca. 30 Ccm. klarer hellgelber Flüssigkeit. Das Zwerch- 
fell steht beiderseits in der Höhe des IV. Intercostalraums, man fühlt anf seiner Abdominalfläche einen böckerigen Ueberzug von kleinen Geschwustknoten. Bei der Entfernung des Brustbeins sieht man an der ganzen Ausdehnung des vorderen Mediastinums zahlreiche linsengrosse flache Knötchen im lockeren Gewebe und längs der beiden Mammariae sitzen, welche meist eine kleine Delle baben, und dadurch grosse Aehnlichkeit mit Pockenpusteln annehmen. Der Berzbeutel enthält wenig klaren wässerigen Inhalt, sein parietales Blatt ist mit einzelnen dieser pockenähnlichen Geschwulstlnnötchen besetzt, wăhrend in dem atrophischen schleimigen Fettgewebe des visceralen Blattes im Umfange beider Vorkammern und der Adventitia der grossen Gefässstämme zahlreiche Eruptionen gleicher Grösse und Form hervortreten. Die Herzhöhlen sind eng, enthalten wenig geronnenes Blut, die Wändeziemlich dick von brauner Farbe und guter Consistenz. Im Septum atriorum sowie im rechten Herzohr fühlt man mehrere flache Verdickungen, welche sich beim Einschneiden als confluirte linsengrosse Tumoren ausweisen. Die Klappen der Aorta sind verdickt, etwas retrahirt, ebenso der freie Rand der Mitralis. Beide L ungen sind voluminös, öberragen mit ihren scharfen Kanten den grösseren Theil des Herzbeutels, sie sind frei beweglich im Thorax, die Pleuraböblen leer. Die Oberflächen sind in den Spitzen blassroth, weiter abwärts dunkelblauroth und mit einem Netzwerk schiefriger bis schwarzer Linien überzogen. An verschiedenen Stellen ist dies Netzwerk sehr dicht und besteht aus weissen etwas erhabenen zarten Strängen, in deren Knotenpunkten linsen- bis haselnussgrosse Tumoren theils der Pleura flach aufsitzen, theils in das Lungenparenchym selbst eindringen. Die Pleura diaphragmatica enthält zahllose flache confluirte Tumoren. Die Bronchialdrüsen sind etwa von Kirschgrösse, schwarz ohne krebsige Einsprengungen. Durchschnitten erweist sich das Lungengewebe durchweg lufthaltig, weich, von mässigem Blutgehalt; die Bronchialschleimbaut blassroth, in den tieferen basalen Abschnitten etwas schleimiges Secret. Längs der Halsgefässe zieht sich beiderseits eine Kette geschwollener Lymphdrüsen bis zur Schädelbasis, die auf der Schnittfläche grauroth aussehen, und mehrfach weisslichen zähen Saft ausdrücken lassen. Die Nervi vagi et phrenici sowie die grossen Stämme des Plexus brachialis sind frei, die Nervenscheiden zart und dünn. Beide Lappen der Schilddrüse sind mässig vergrössert, enthalten zahllose kleine Gallertcystchen. Die Schleimhaut der Rachenorgane des Kehlkopfs und der Trachea ist blass, sonst unverändert. Die Milz ist. klein, ihre Kapsel diffus verdickt und trübe, das Parenchym ziemlich derb, mit deutlichen Trabekeln.

Beide Nieren sind etwas verkleinert, sie enthalten in der Fettkapsel und der Albuginea zahlreiche flache, rundliche Geschwülste von höchstens 10 Markstückgrösse, dagegen finden sich in der übrigens etwas schmalen und feingranulirten Rinde nur wenige Knötchen, welche kaum den Umfang eines Stecknadelkopfes übersteigen. Die Marksubstanz ist anffallend geröthet. Beide Nebennieren sind sebr stark geschwollen, $7 \mathrm{Cm}$. lang, 3 breit, $2-3$ dick; sie fühlen sieh derb an, und ergeben auf dem Durchschnitt eine so dichte Eruption meist erbsengrosser grauweisser Tumoren in der Rinde, dass diese scheinbar eine zusammenhängende $1 \mathrm{Cm}$. dicke Zone bilden, innerhalb deren die bräunliche Intermediärschicht und die graue 
Markmasse noch vielfach deutlich erkennbar sind. Die Blas e enthält reichlich klaren hellen Urin. Ihre Schleimhaut ist blassgrau, sie enthält etwa 10 durchaus pockenähnliche erhabene Krebsknoten, welche nach dem Blasenhalse zu mehrfach confluiren, und oberfächlich hie und da schwache Incrustationen ron Harnsalzen erkennen lassen. Beim Einschneiden ergiebt sich, dass sie in der Mucosa selbst ihren Sitz haben, die tieferen Wandschichten sind frei davon. Beide Eierstöcke sind fast von der Grösse eines Gänseeies, die Kapseln verdickt und lederartig derb, grauweiss. Auch hier beruht die Vergrösserung wie bei den Nebennieren auf einer Einsprengung zahlreicher haselnussgrosser, confluirter Tumoren in die Randzone der Organe. Die centralen Gewebsabschnitte sind in eine weiche beinahe myxomatös aussehende, wässerig durchtränkte Sulze umgewandelt. Uterus klein, mit verdickten Gefässen der Wand, sonst intact. Im Duodenum galliger Inhalt, die Innenfläche normal. Der Magen ist mässig welt, dünnwandig, blassgrauweiss, nahe dem Pylorus an der grossen Curvatur liegen 6 flache derbe Tumoren, deren grösster Zehnmarkstückumfang erreicht, während die übrigen 5 nur linsengross sind. Ihr Rand ist um $2 \mathrm{Mm}$. über das umliegende Schleimbautniveau erhaben, das Centrum etwas abgeflacht, nicht ulcerirt. Wie der Durchschnitt lehrt, reichen sie nicht unter die Submucosa in die Tiefe. Die Leber ist braun atrophisch, enthält an ihrer Oberfläche einen zweimarkstückgrossen, im Centrum nabelartig eingezogenen Tumor, und ausserdem mehrere kirschgrosse, rein weisse markige Knoten im Innern. Der Darm ist frei von Neubildungen, dagegen sind die mesenterialen, die lumbalen, retroperitonealen und epigastrischen Lymphdrüsen, sowie der Strang, welcher im hinteren Mittelfellraum verläuft, geschwollen und degenerirt. Der Ductus thoracicus ist eng, seine Wand dünn und durchscheinend, er enthält klare Lymphe. Die Aorta weit, dickwandig, Intima glatt.

Das Scbädeldach ist sehr dick, beide Tafeln des Knochens von elfenbeinerner rein weisser Knochensubstanz, Oberfläche glatt, die Diploë von mittlerem venösen Blutreichthum. Die Dura ist schlaff, darchscheinend, dünn, im Längssinus spärliches Gerinnsel; ihre Innenfläche ist rein weiss, trocken. Auch die Pia ist auffallend trocken, ihre Gefässe beinahe leer, die Convexität transparent und zart. An der Basis bestebt eine ziemlich starke Verdickung und Trübung ihres Gewebes, rechts in der hinteren Schädelgrube eine circumseripte Verwachsung mit der Dura. Hier bemerkt man einen reichlich kirschgrossen derben graugelben Geschwulstknoten, welcher an der Austrittsstelle des rechten Facialis und Acusticus liegt, und diese Nerven bis zur Unkenntlichkeit umwachsen hat. Der Knoten líegt dem Kleinhirnstiel unmittelbar auf, und hat sich gegen Pons und Cerebellom eine kleine Delle eingedrückt, ohne die Piadecke derselben zu durchbrechen. Auf derselben Seite enthalten noch der Abducens, der Trigeminus, der Vagus und Glossopharyngeus hart an ihrem Austritt aus dem Gehirn knollige, sehr derbe Infiltrationen, durch welche sie 1,2 oder 3 erbsengrosse Anschwellungen erhalten, die sich belm Einschneiden als Krebsknötchen ausweisen. Links zeigen mit auffallender Symmetrie der Trigeminus und Vagus ebensolche derben, noch etwas kleineren, kugeligen Auftrelbungen. Die Gehirnsubstanz ist von geringem Blutgehalt, die Ventrikel weit, mit reichlichem Liquor, weitere Metastasen nicht nachwetsbar. 
Die Pia des Rückenmarks ist in ganzer Ausdehnung verdickt, trübe, ödematös, entbält reichliche Knochenplättchen eingestreut. Die Medulla selbst ist blass und unverändert. Die Nervenwurzeln und zwar die vorderen wie die hinteren entbalten grossentheils bart an jbrem Austritt aus dem Rückenmark erbsengrosse kugelige Geschwulstknöpfchen, durch welche der scheinbar intacte Nerv mitten bindurchtritt. In der Cauda equina werden diese Knoten so zahlreich, dass auf manchen Nervensträngen 5,6 und mehr Tumoren wie Perlen auf einer Schnur aufsitzen, und dass kaum ein einziger Nerv ganz frei von Neublldungen ist. Nach ibrem Durchgange durch die Intervertebrallöcher sind die Nerven durchaus normal und ohne krebsige Veränderungen.

Mikroskopisch enthatten sämmtliche Metastasen ein Krebsgewebe mit kleinen zartwandigen Alveolen und sehr reichlichen niedrigen, cubischen Zellen, welche die Grösse von Milchdrüsen epithelien nirgends wesentlieh überschreiten. Im Gehirn, und namentlich an den Metastasen der Nervenwurzeln lässt sich sehr deutlich das Wuchern der Neubildung im Bindegewebe der Pia verfolgen, von dem aus man auf Querschnitten aussen breite, nach innen sich zuspitzende Zellenzapfen erkennt, welche von der Nervenscheide in den intrafasciculären Spalten centripetal vordringen, und ihrer Ausbreitung entsprechend, die Nerven zum Schwunde bringen. Die eigenthümlichen nabelähnlichen Dellen in den kleineren Eruptionen des Herzbeutels, des Mediąstinums und der Harnblase beruhen auf Fettmetamorphosen in Stroma und Zellen. In einzelnen Knötchen der Cauda bemerkt man Concretionen von Hirnsand. -

Fassen wir nun diese 4 Fälle mit demjenigen Cohnheim's, den beiden multiplen submucösen Metastasen bei Carcinoma oesophagi von Petri und einem von Weigert dieses Archiv Bd. 67 beschriebenen Fall von Magenmetastasen nach einem krebsigen Unterschenkelgeschwür zu einer kleinen Statistik zusammen, so ergiebt sich, 1) dass $4 \mathrm{mal}$ primäre Carcinome des Oesophagus, 2mal primäre Careinome der Mamma, 1mal primäres Carcinom des Hodens und $1 \mathrm{mal}$ primäres Carcinom des Unterschenkels den Ausgang für Metastasen des Magens bildeten. 2) Dass der Magen in 2 Fällen nur einen, in den übrigen 6 Fällen zwei und mehrere secundäre Tumoren enthielt. 3) Dass dieselben in 2 Fällea alle Schichten der Magenwand durehsetzten, während 6 mal nur Mucosa und Submucosa betroffen waren. 4) Dass weitere Veränderungen solcher Krebsgeschwülste durchaus nicht ausgeschlossen sind, wie Förster angiebt, dass vielmehr in 4 Fällen Ulceration ganz analog den Pri- 
märkrebsen eingetreten ist. 5) Dass in allen 8 Fällen die Neubildung einen circumscripten Charakter an sich trug.

Dieses letzte Merkmal scheint also bei aller Mannichfaltigkeit in der äusseren Erscheinung einen constanten Factor darzustellen, welcher in zweifelhaften Fällen für die Frage nach der primären oder secundären Natur des Carcinoms entscheidend wäre.

\title{
VII.
}

\section{Verbreitung des Kropfes und Cretinismus im Kaukasus.}

\author{
Von N. v. Seidlitz, \\ Oberredacteur des Kaukasischen Statistischen Comite's in Tiflis.
}

(Hierzu Taf. V.)

Am 5./17. März 1858 berichtete Karl Ernst von Baer in der Kaiserlichen Akademie der Wissenschaften zu St. Petersburg ${ }^{1}$ ), in Veranlassung einer Anfrage des durch seine Versuche, den Cretinismus im Institut zu Abendberg (Canton Bern) zu heilen, bekannten Dr. Guggenbühl, über das dazumal bekannte Vorkommen jenes Leidens im Russischen Reiche. Vom Kaukasus wusste unser bedeutender Anthropologe damals in dieser Beziehung nichts zu sagen und scheinen auch die bezüglichen officiellen Anfragen von keinem Erfolge gewesen zu sein.

Und doch hatte vor schon anderthalbhundert Jahren der griechische Geograph, Zarewitsch Wahuscht, beiläufig der Existenz des Kropfes im Kaukasus Erwăhnung gethan. Uıständlichere Berichte über dieses Leiden begannen in den 50 ger Jabren des laufenden Jahrhunderts aufutauchen. Der selige General Bartholomåi, der seine Excursion nach Sswanethien im Sommer 1853 ausführte, wies auf das Vorhandensein des Kropfes in jener Gegend hin $^{2}$ ). Bald darauf theilten Notizen uber die Existenz des Kropfes, wie in

1) Mélanges Biolog. tírés du Bull. phys.-mathém. de l'Acad. Imp. des Sc. de St. Petersb. 1858. S. $634-646$.

2) Reise nach Sswanethien. Sapiskl (Mémoiren) der kauk. Section der Kais. Russ. Geogr. Ges. B. III. S. 32, 59 und 61 (russ.). 